\title{
Modelo de producción social de habitat frente al modelo de mercado en la construcción de vivienda de interés social
}

\section{Habitat social production model in front of market model in social housing construction}

\section{Carlos Fernando Agudelo Rodríguez}

Arquitecto, magíster en Planificación Urbana y Regional. Docente Asistente de la Universidad de la Salle. Bogotá, Colombia.

Contacto: cfagudelo@unisalle.edu.co.

\section{Martha Lucía Vaca Bohórquez}

Psicóloga, MBA. Consultora e investigadora de la Universidad de los Andes. Bogotá, Colombia. Contacto: ml.vaca68@uniandes.edu.co.

\section{César Augusto García Ubaque,} Ingeniero Civil, doctor en Ingeniería. Docente de la Universidad Distrital Francisco José de Caldas. Bogotá, Colombia. Contacto: cagarciau@udistrital.edu.co.

Palabras clave: construcción, organización comunitaria, planificación urbana, viviendas.

Keywords: community organizing, construction, housing, urban planning.

\section{RESUMEN}

El presente artículo presenta los resultados de la investigación que analiza dos de los principales modelos que se aplican actualmente en Bogotá para atender las necesidades de vivienda de las familias de bajos ingresos -mercado y Producción Social de Hábitat y Vivienda (PSHV)-. Se construyó un marco conceptual fundamentado en la definición de los términos de vivienda y hábitat a la luz de nuevos enfoques de desarrollo. Se realizó una caracterización de los modelos de producción de VIS y se diseñó y aplicó una metodología para el análisis de sus procesos y resultados. Lo anterior permitió plantear algunas conclusiones que apuntan hacia el reconocimiento del modelo de PSHV como una variante del modelo de mercado, teniendo en cuenta que las iniciativas para el desarrollo de soluciones habitacionales se concretan de forma muy similar en ambos casos. 


\section{ABSTRACT}

The objective of this research was to analyze two social housing construction models that are currently applied in low income families of Bogotá (Habitat social production 'HSP and market models). We built a framework based on housing and habitat concepts according to recent development approaches. We characterized these construction models and designed a methodology, which was applied to analyze processes and results for each case. We concluded that, although HSP model is a variant of market model, the process for giving housing solutions were similar in both cases.

\section{INTRODUCCIÓN}

El presente artículo muestra los resultados del proyecto Análisis de modelos para producción de vivienda de interés social en Bogotá [1] que tuvo como objetivo analizar la efectividad de dos tipos de ofertas de vivienda formal que se han ejecutado en la ciudad de Bogotá (Colombia), para dar respuesta a las necesidades de familias de bajos ingresos. Estos modelos son: el que lleva a cabo el sector privado y el ofrecido por organizaciones sociales sin ánimo de lucro. Se buscó también proponer elementos de juicio que faciliten la definición de futuras estrategias por parte del Estado, la sociedad civil y organismos internacionales.

De manera específica, se caracterizó cada uno de los dos modelos en términos de: habitabilidad familiar y comunitaria, se compararon los resultados de ambos modelos y se evaluaron los procesos generados en la organización y en la autogestión comunitaria.

Como hipótesis de trabajo se planteó que la producción social de hábitat y vivienda (PSHV) ofrece productos con mejores condiciones de habitabilidad y accesibilidad para las familias de bajos ingresos, con lo cual se contribuye, en mayor medida, al desarrollo de familias y comunidades, frente a las alternativas ofrecidas por la vía de mercado. Se asumió que los dos modelos de oferta de vivienda tienen similitudes en cuanto a condiciones de calidad, tanto para el entorno de la 


\section{investigación}

Esta matriz propone nueve necesidades axiológicas -o de valores- que son: subsistencia, protección, afecto, entendimiento, participación, ocio, creación, identidad y libertad; y para cada una de ellas, establece satisfactores clasificados en las categorías existenciales de: ser, tener, hacer y estar. A manera de ejemplo, la tabla 1 muestra los satisfactores propuestos para la necesidad axiológica de subsistencia.

Dentro de este contexto, la vivienda y el hábitat se entienden como bienes que afectan la eficiencia de los satisfactores de abrigo y entorno vital, por lo cual, son aspectos que se relacionan con las categorías esenciales de ser y estar y su satisfacción genera un aporte frente a la necesidad de subsistencia. Adicionalmente, Sen [4] considera que el desarrollo de una sociedad debe ser entendido como la expansión de las libertades reales de sus individuos, por lo que la pobreza, la tiranía y la escasez de oportunidades económicas, se constituyen las principales fuentes de privación de libertad y, en consecuencia, la tenencia de una vivienda adecuada es una libertad fundamental y un elemento clave en el proceso de desarrollo de capacidades de los individuos o, como lo plantea UN-HÁBITAT, una dotación inicial que es el patrimonio con el que cuentan las personas para poner en acción sus capacidades, las cuales le permiten ejercer unos derechos que se traducen en un determinado nivel de vida [5].

Por su parte, la Organización de Naciones Unidas (ONU) dentro de su enfoque de derechos y libertades, considera a la vivienda como un bien al cual se accede mediante el aumento del nivel de vida: "Toda persona tiene derecho a un nivel de vida adecuado que le asegure, así como a su familia, la salud y el bienestar, y en especial la alimentación, el vestido, la vivienda, la asistencia médica y los servicios sociales necesarios" [6] y específicamente en Colombia, la Constitución Política de 1991 determina que "Todos los colombianos tienen derecho a vivienda digna. El Estado fijará las condiciones necesarias para hacer efectivo este derecho y promoverá planes de vivienda de interés social, sistemas adecuados de financiación a largo plazo y formas asociativas de ejecución de los programas de vivienda" (art. 51).

Tomando en cuenta lo anterior, para fines de la investigación desarrollada, se entiende la vivienda como un bien de derecho, para cuyo acceso, los habitantes deberán contar con el apoyo del gobierno a través de la implementación de planes y programas, y se resaltan como elementos importantes, por un lado: la acción comunitaria y la asociatividad, que permiten que la vivienda se constituya como un componente de organización social; y por otro, la autogestión como un instrumento alternativo de materialización de este derecho.

\subsection{Concepto de vivienda}

De acuerdo con la ONU, una vivienda adecuada significa disponer de un lugar donde poder aislarse si se desea, con espacio y seguridad adecuada,

Tabla 1. Satisfactores para la necesidad de subsistencia

\begin{tabular}{|c|l|l|l|l|}
\hline Necesidad & \multicolumn{1}{|c|}{ Ser } & Tener & Hacer & Estar \\
\hline & Salud física & Alimentación & Alimentar & Entorno vital \\
& Salud mental & Abrigo & Procrear & Entorno social \\
Subsistencia & Equilibrio & Trabajo & Descansar & \\
& Solidaridad & & Trabajar & \\
& Humor & & & \\
& Adaptabilidad & & & \\
\hline
\end{tabular}

Fuente: tomado de [3] 
iluminación y ventilación e infraestructura básica adecuadas y una ubicación apropiada en relación con el lugar de trabajo y servicios básicos. Todo ello a un costo razonable [7]. Esta definición, permite establecer si una vivienda es o no una solución válida, según sus características físicas internas -funcionalidad y confort-, su dotación de infraestructura básica -servicios públicos, vías, transporte y equipamiento social- y su localización -urbana, rural-. Adicionalmente, una vivienda debe garantizar que el grupo familiar pueda desarrollar procesos de interrelación entre sus miembros y también permitir que cada uno de sus miembros pueda realizar actividades personales, según sus tradiciones culturales.

Por su parte, la vivienda de interés social VIS se constituye en un capítulo dentro de este concepto general, enfocado hacia los grupos sociales que, debido a sus bajos ingresos, no pueden adquirir vivienda a través de mecanismos netamente comerciales. Este tipo de vivienda se define como un bien de mérito no mercantil, convenida socialmente como responsabilidad del Estado y cuya privación afecta las dotaciones iniciales, el desarrollo de capacidades y el nivel de calidad de vida [5]. Los bienes meritorios son aquellos que, aunque en rigor son de tipo privado -su consumo se agota en quien lo recibe individualmente-, el efecto de este consumo, excede en términos sociales el beneficio del consumidor individual. Son bienes que son privilegiados por la sociedad porque promueven el desarrollo de capacidades y nivel de vida de toda la sociedad como un conjunto.

En este mismo sentido, para el caso de Colombia, la Ley 388 de 1997 (art. 91) concibe la vivienda de interés social como la que se ofrece para garantizar el derecho que a ella tienen los hogares de menores ingresos. De acuerdo con lo anterior, se puede afirmar que la vivienda no solo puede entenderse como una mercancía o producto que satisface una necesidad básica, sino que además tiene una función fundamental como condición para el desarrollo de las capacidades de los seres humanos y como un bien indispensable para la elevación de las condiciones de vida de los individuos y sus familias, especialmente los más pobres.

\subsection{Hábitat, más allá de la vivienda}

¿Es la vivienda, en tanto elemento construido o adquirido individualmente, capaz de mejorar, por si sola, las condiciones de vida de sus ocupantes? Frente a esta pregunta, es importante tener en cuenta los elementos que componen la interioridad del lugar para vivir, pero al mismo tiempo deben involucrarse elementos propios de los entornos próximos y remotos [8]. El hábitat es soporte y condición, al tiempo que es espacio resignificado y reconstruido por la cultura [9], así como, según lo plantea la organización Hábitat International Coalition (HIC) el conjunto de condiciones ambientales y materiales que permiten la satisfacción de las necesidades vitales y la supervivencia de una especie. Este hábitat está determinado además por factores económicos, sociales, culturales y políticos que facilitan o limitan el acceso de todos a los bienes y servicios que la sociedad produce [10].

En Colombia, los procesos de urbanización y construcción no se han ajustado a esta lógica y entre un $20 \%$ y un $50 \%$ de las ciudades colombianas han sido construidas informal y espontáneamente [11]. Estos procesos, además de producir un déficit cuantitativo, que de acuerdo con los datos más recientes del DANE, para el 2005 era de 623078 soluciones de vivienda (12\%) en las trece áreas metropolitanas del país [12], no han resuelto y por el contrario han deteriorado la calidad de la vivienda disponible, en variables como: disponibilidad de servicios públicos -agua, luz, alcantarillado-, existencia de baño y cocina y hacinamiento -para ese mismo año el DANE calculó un déficit cualitativo de 456681 viviendas (9\%)-. 


\section{investigación}

Algunos de los factores que coadyuvan a esta situación son: la dificultad para vincular los hogares de menores ingresos al sistema financiero, la especulación con el precio del suelo, la escasez de tierra urbanizable, la reducida oferta privada de VIS y la ineficacia de las políticas de vivienda planteadas por el gobierno.

En la presente investigación se realizó una comparación entre dos de las principales alternativas que se han abordado para proveer vivienda a los grupos sociales de menores ingresos en la ciudad de Bogotá. Estas son: oferta del mercado, que se refiere a la producción efectiva de viviendas por parte de empresas de tipo capitalista que ofrecen abiertamente sus productos a una demanda dispersa; y la segunda, de producción social de hábitat y vivienda PSHV, promovida por algunas ONG que desarrollan procesos colectivos, participativos y autogestionarios de producción de vivienda en los que recogen algunos de los aspectos constitutivos de los procesos históricos de autoconstrucción.

\subsection{Producción de vivienda a través del mercado}

Esta alternativa supone que el mercado ofrecerá a la sociedad los bienes que ella necesite o demande y sobre este aspecto, desde 1990, el país maneja un programa de subsidio a la demanda para la adquisición de vivienda denominado Subsidio Familiar de Vivienda de Interés Social, el cual delega en los productores la responsabilidad de solucionar el problema de déficit de Vivienda de Interés Social. Son las empresas constructoras las que diseñan soluciones habitacionales con base en la aplicación de modelos comerciales que garanticen utilidades sobre su inversión. Este proceso se caracteriza por: a) los usuarios no intervienen -al menos directamente- en la etapa de diseño, b) se requiere que las familias interesadas cumplan con los requisitos de estabilidad de in- gresos y capacidad de endeudamiento del sistema bancario, c) los beneficiarios están excluidos del proceso de construcción, d) las familias pueden acceder al subsidio familiar de vivienda que otorga el gobierno nacional, previo cumplimiento de los requisitos establecidos en la ley; y por último, e) se utilizan sistemas constructivos industrializados que garantizan agilidad y optimización de costos.

\subsection{Producción de vivienda por PSHV}

La producción social de hábitat y vivienda, se fundamenta en la posibilidad de que las familias y comunidades organizadas participen en la producción de sus propias viviendas y hábitat, de manera que controlen las decisiones fundamentales del proceso [13]. La Hábitat International Coalition (HIC), la define como un conjunto de experiencias y alternativas que centran la producción, acceso a la vivienda y a la ciudad, en la gente, sus necesidades, sus formas de vida, anhelos y organización social. Es especialmente efectivo cuando se apoya en procesos autogestionarios colectivos que incluyan capacitación, participación responsable, organización y solidaridad activa entre los participantes. De esta manera se contribuye a fortalecer prácticas comunitarias, al ejercicio directo de la democracia, la autoestima de los participantes y una coexistencia social más activa. Ortiz [14], presidente de la HIC, afirma que la PSHV no solo es generadora de paredes y techos, sino también de ciudadanía consciente, productiva y responsable y a la vez se constituye en un potenciador de la economía popular.

Para que este sistema sea efectivo se requiere que existan actores participativos y proclives a la participación, planificación flexible, un diagnóstico surgido de necesidades comunitarias concertadas, decisiones tomadas participativamente, un plan de construcción y proyectos que expresen lo posible, sobre la base del consenso y el conflicto 
[13]. Debe tener como ejes básicos: el desarrollo organizacional de los participantes, la interacción con el Estado y la posibilidad de diseñar reglas propias para la construcción [15].

En Colombia, algunas ONG han diseñado modelos específicos de intervención dentro del concepto de PSHV que se han caracterizado por: la constitución legal de una asociación o figura similar por parte de la comunidad beneficiaria, la organización de un plan de ahorro para asegurar los recursos requeridos y acceder al subsidio familiar de VIS, la obtención de créditos complementarios ofrecidos por la ONG, ya sea directamente o a través de un intermediario financiero. En todos los casos, también debe ofrecer capacitación en temas que aporten al fortalecimiento y desarrollo de capacidades tanto en el plano individual como colectivo, presencia de actividades generadoras de ingresos, junto con una marcada tendencia hacia la inclusión de actividades de grupos especiales -niños, jóvenes y ancianos- y la participación activa de la comunidad en las fases de diseño, producción de materiales y construcción.

\section{METODOLOGÍA}

Para este estudio, la unidad de análisis estuvo constituida por proyectos habitacionales realizados en la ciudad de Bogotá, tanto por empresas privadas mediante la aplicación de modelos comerciales, como por ONG mediante modelos fundamentados en el concepto PSHV. Se analizaron tres proyectos en cada modalidad.

El análisis realizado fue de tipo evaluativo, en términos de la pertinencia y relevancia frente a su contribución al logro de los objetivos sociales de provisión de hábitat para grupos de bajos ingresos, fue una evaluación ex-post-después de realizados los proyectos- y orientada a revisar de manera comparativa, por personal externo no vinculado a los proyectos analizados.
Se utilizó una metodología de estudio de caso comparativo, que se define como un examen intensivo -tanto en amplitud como en profundidad- de cada unidad de estudio [16] y los datos resultantes se ordenaron de manera que el carácter unitario de cada muestra fuera preservado.

\subsection{Matriz de organización analítica de resultados}

Se utilizó una matriz que operacionaliza los temas y dimensiones de análisis requeridos en esta investigación [17]. Su estructura es jerárquica a partir de la desagregación de los temas fundamentales por evaluar, pasando de un nivel macro al micro y llegando hasta el nivel de las variables. Esta matriz se compone de: dimensiones, subdimensiones, aspectos y variables, organizadas de acuerdo con la figura 1.

Las dimensiones son las grandes aproximaciones a la habitabilidad desde la perspectiva de la familia y de la comunidad que son afectadas por el proyecto habitacional, y para este caso, se abordaron tres dimensiones: condiciones familiares

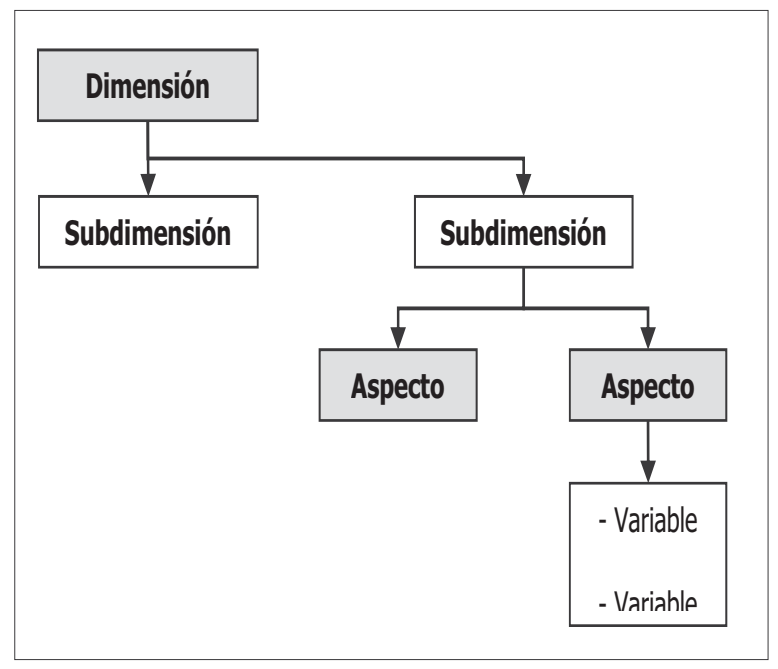

Figura 1. Estructura matriz de evaluación

Fuente: tomado de [17]. 


\section{investigación}

de habitabilidad, condiciones comunitarias de habitabilidad y organización y autogestión comunitaria -solo para proyectos de PSHV-.

Las subdimensiones son los componentes en los que se desagregan las dimensiones de análisis y en las cuales se deberían producir los resultados como consecuencia de la ejecución del proyecto habitacional. Estas subdimensiones se operacionalizaron en aspectos o formas de desagregación de los elementos conceptuales que permiten aproximarlos a la realidad. Las variables son aspectos de la realidad que cambian en el tiempo y de un proyecto a otro. Todos estos componentes fueron examinados mediante indicadores cuantificables. En la tabla 2, se presenta la estructura de los elementos incluidos en la matriz utilizada.

Los instrumentos utilizados para la recolección de la información necesaria para evaluar los proyectos fueron:

a) Fichas de recolección de información secundaria: permitieron sistematizar la información proveniente de fuentes secundarias como: planos, reglamentos de propiedad horizontal, escrituras de propiedad, y otros documentos oficiales.

b) Encuestas: fueron utilizadas para recolectar información de fuentes primarias: nivel familiar.

c) Entrevistas semiestructuradas y grupos focales: se utilizaron para recolectar información de fuentes primarias a nivel comunitario o barrial.

\subsection{Proyectos seleccionados}

La selección de las unidades de análisis se efectuó a partir de varios criterios que garantizaran su comparabilidad, principalmente con el tipo de destinatarios y la pertenencia a una misma localidad.

Los seis proyectos habitacionales, objeto de estudio de la presente investigación, están ubicados

Tabla 2. Elementos considerados en la matriz de análisis

\begin{tabular}{|c|c|c|c|}
\hline Dimensiones & Subdimensiones & Aspectos & Variables \\
\hline \multirow{6}{*}{$\begin{array}{l}\text { Condiciones } \\
\text { familiares } \\
\text { de habitabili- } \\
\text { dad }\end{array}$} & Tenencia & & $\begin{array}{l}\text { Tipo de tenencia } \\
\text { Título de tenencia }\end{array}$ \\
\hline & \multirow{4}{*}{ Características } & Área y diseño & $\begin{array}{l}\text { Dimensiones del lote, área de la vivienda, área de cons- } \\
\text { trucción por persona y percepción familiar del tamaño }\end{array}$ \\
\hline & & $\begin{array}{l}\text { Construcción, adiciones y } \\
\text { calidad }\end{array}$ & $\begin{array}{l}\text { Calidad de muros, placas y cubiertas, posibilidades de } \\
\text { ampliación en altura, área de planta y espacios internos }\end{array}$ \\
\hline & & $\begin{array}{l}\text { Instalaciones y servicios in- } \\
\text { ternos }\end{array}$ & $\begin{array}{l}\text { Existencia y provisión de agua potable, energía eléctri- } \\
\text { ca, gas natural, telefonía fija y desagües }\end{array}$ \\
\hline & & Condiciones de confort & $\begin{array}{l}\text { Iluminación natural y artificial, ventilación, aislamiento } \\
\text { térmico y aislamiento acústico }\end{array}$ \\
\hline & $\begin{array}{l}\text { Costos y } \\
\text { financiamiento }\end{array}$ & & $\begin{array}{l}\text { Costo de la vivienda, forma de pago, medios de finan- } \\
\text { ciamiento (tipo y condiciones) y accesibilidad de valor de } \\
\text { cuotas (amortización / arrendamiento) }\end{array}$ \\
\hline
\end{tabular}




\section{investigación}

\begin{tabular}{|c|c|c|c|}
\hline Dimensiones & Subdimensiones & Aspectos & Variables \\
\hline \multirow{13}{*}{$\begin{array}{l}\text { Condiciones } \\
\text { comunitarias } \\
\text { de habitabili- } \\
\text { dad }\end{array}$} & $\begin{array}{l}\text { Localización } \\
\text { de la comunidad }\end{array}$ & & $\begin{array}{l}\text { Accesibilidad a actividades básicas (trabajo, estudio, co- } \\
\text { mercio, servicios zonales), accesibilidad desde y hacia } \\
\text { zonas vecinas, características topográficas, condiciones } \\
\text { de riesgo físico y condiciones de amenaza ambiental }\end{array}$ \\
\hline & \multirow{9}{*}{$\begin{array}{l}\text { Servicios } \\
\text { públicos }\end{array}$} & Agua potable & \multirow{7}{*}{$\begin{array}{l}\text { Disponibilidad, red interna, conexión a redes matrices, } \\
\text { ente prestador y calidad }\end{array}$} \\
\hline & & Energía eléctrica & \\
\hline & & Gas de consumo doméstico & \\
\hline & & Desagüe de aguas negras & \\
\hline & & Desagüe de aguas lluvias & \\
\hline & & Telefonía fija domiciliaria & \\
\hline & & Alumbrado público & \\
\hline & & Recolección de basuras & Disponibilidad en la zona, ente prestador y calidad \\
\hline & & Transporte público & Transporte más utilizado y calidad \\
\hline & Infraestructura vial & & $\begin{array}{l}\text { Existencia, perfil, consolidación y estado de vías peato- } \\
\text { nales y vehiculares internas }\end{array}$ \\
\hline & $\begin{array}{l}\text { Desarrollo } \\
\text { urbanístico }\end{array}$ & & $\begin{array}{l}\text { Cumplimiento de la normatividad urbana, tipo de loteo, } \\
\text { implantación de las viviendas, ocupación de lote, densi- } \\
\text { dad de vivienda, configuración de manzanas o bloques }\end{array}$ \\
\hline & $\begin{array}{l}\text { Servicios } \\
\text { sociales }\end{array}$ & & $\begin{array}{l}\text { Disponibilidad, uso y calidad de servicios de: seguridad, } \\
\text { salud y educación }\end{array}$ \\
\hline \multirow{4}{*}{$\begin{array}{l}\text { Organización } \\
\text { y autogestión } \\
\text { comunitaria }\end{array}$} & \multirow{2}{*}{$\begin{array}{l}\text { Desarrollo } \\
\text { organizacional }\end{array}$} & $\begin{array}{l}\text { Características de la orga- } \\
\text { nización }\end{array}$ & $\begin{array}{l}\text { Momento de conformación, motivación, tipo de organi- } \\
\text { zación, nivel de formalización, composición (estructura } \\
\text { interna y cantidad de integrantes) y democracia interna } \\
\text { (modo de elección de la dirigencia, formas de divulgación } \\
\text { de la información, y formas de toma de decisiones) }\end{array}$ \\
\hline & & $\begin{array}{l}\text { Definición de objetivos, pro- } \\
\text { gramas y organización }\end{array}$ & $\begin{array}{l}\text { Identificación colectiva y priorización de necesidades, } \\
\text { definición de objetivos comunes, definición de alternati- } \\
\text { vas de solución, diseño de la estrategia de solución y } \\
\text { organización para la implementación de la estrategia de } \\
\text { solución }\end{array}$ \\
\hline & \multirow[t]{2}{*}{$\begin{array}{l}\text { Desempeño } \\
\text { organizacional }\end{array}$} & Relación con otros actores & $\begin{array}{l}\text { Reconocimiento de la importancia de vínculos con otros } \\
\text { actores (públicos y privados), identificación de otros ac- } \\
\text { tores relacionados, formalización de vínculos con otros } \\
\text { actores, tipo de apoyo recibido, dinámica de trabajo con- } \\
\text { junto }\end{array}$ \\
\hline & & $\begin{array}{l}\text { Aporte organizativo a la } \\
\text { gestión del proyecto }\end{array}$ & $\begin{array}{l}\text { Aporte de la organización en las fases de formulación, } \\
\text { ejecución y seguimiento del proyecto habitacional y pro- } \\
\text { ceso de ocupación }\end{array}$ \\
\hline
\end{tabular}

Fuente: elaboración propia

44 Tecnura | Vol. $17 \mid$ No. 38 | Octubre - Diciembre de 2013 


\section{investigación}

Tabla 3. Proyectos habitacionales seleccionados

\begin{tabular}{|c|l|l|l|l|}
\hline No. & \multicolumn{1}{|c|}{ Proyecto habitacional } & \multicolumn{1}{|c|}{$\begin{array}{c}\text { Modelo } \\
\text { de producción }\end{array}$} & $\begin{array}{c}\text { Número de } \\
\text { soluciones }\end{array}$ & Promotor \\
\hline 1 & Quintas del Recreo Etapa 4 & Mercado & 248 & Urbe Capital \\
\hline 2 & Alameda de Santa Mónica Etapa 5 & Mercado & 237 & MARVAL \\
\hline 3 & Kasay de Los Venados Etapa 2 & Mercado & 270 & Diseño Urbano \\
\hline 4 & Renacer & PSHV & 168 & OPV Renacer \\
\hline 5 & Nueva Ciudad Etapa 1 & PSHV & 177 & OPV Nueva Ciudad \\
\hline 6 & Casa Grande Etapa 1 & PSHV & 230 & Asociación de Vivienda Popular AVP \\
\hline
\end{tabular}

Fuente: elaboración propia

en la Ciudadela El Recreo, barrio localizado en la UPZ 87 (Tintal Sur) de la localidad de Bosa. La Ciudadela El Recreo, se constituyó en 1999 como el primer proyecto de ejecución directa de Metrovivienda y tiene una extensión de 115,38 hectáreas. Su diseño urbanístico contempla 32 manzanas, de aproximadamente una hectárea cada una, para desarrollo de proyectos de VIS y cada cuatro manzanas se conforma un espacio público destinado a servicios comunitarios. Cuenta con áreas comerciales, $1,8 \mathrm{~km}$ de parque lineal y espacio público que conecta a toda la ciudadela por vías peatonales y ciclorutas, además está dotada de cinco comedores comunitarios, cinco colegios, y más de diez jardines infantiles, entre otros servicios. En la tabla 3 se presentan los seis proyectos y el modelo de producción al que pertenece cada uno.

\section{RESULTADOS}

Las tablas 4, 5 y 6 muestran los resultados obtenidos a través de la aplicación de los instrumentos seleccionados.

Se observa similitud entre estas dos modalidades de producción debido a que aún en los proyec- tos producidos por PSHV no hubo participación activa de los beneficiarios. Las características de las soluciones ofrecidas tanto en configuración física, como en la manera de adquirirlos, fueron definidas por los responsables de las $\mathrm{ONG}$, sin la participación de los futuros usuarios. Aún en los procesos de PSHV -por lo menos en los casos analizados- se presenta una separación entre el rol de productor y de consumidor, similar a la de la modalidad del mercado.

El tema comunitario en los procesos de PSHV fue tratado de una manera formal -cursos y talleres sobre temas de organización de beneficiarios-, pero no hizo parte de manera real del proceso de solución. Únicamente se realizaron actividades de organización de demanda, complementados con capacitación básica sobre el proyecto habitacional.

Esta capacitación tenía como objetivo principal cumplir los requisitos exigidos y facilitar el acceso de los interesados a créditos para financiar la compra de sus viviendas.

De acuerdo con lo observado, en estos proyectos, el modelo de PSHV tiene características que 


\section{investigación}

Tabla 4. Resultados obtenidos de la Dimensión 1

\begin{tabular}{|c|c|c|c|c|c|c|}
\hline \multicolumn{3}{|c|}{$\begin{array}{c}\text { Dimensión 1. Condiciones familiares } \\
\text { de habitabilidad }\end{array}$} & \multicolumn{2}{|c|}{$\begin{array}{l}\text { Modelo Mercado } \\
(\%)\end{array}$} & \multicolumn{2}{|c|}{$\begin{array}{l}\text { Modelo PSHV } \\
(\%)\end{array}$} \\
\hline \multirow{2}{*}{\multicolumn{2}{|c|}{ Tenencia }} & Propietarios & \multicolumn{2}{|c|}{93,88} & \multicolumn{2}{|c|}{87,88} \\
\hline & & $\begin{array}{l}\text { Título (Escritura pú- } \\
\text { blica) }\end{array}$ & \multicolumn{2}{|c|}{79,59} & \multicolumn{2}{|c|}{80,30} \\
\hline \multirow{17}{*}{ Características } & \multirow{3}{*}{ Área y diseño } & \multirow{3}{*}{$\begin{array}{l}\text { Percepción familiar } \\
\text { del tamaño }\end{array}$} & Grande & 8,16 & Grande & 13,64 \\
\hline & & & Adecuada & 55,10 & Adecuada & 57,58 \\
\hline & & & Pequeña & 36,74 & Pequeña & 28,78 \\
\hline & \multirow{4}{*}{$\begin{array}{l}\text { Construcción, adi- } \\
\text { ciones y calidad }\end{array}$} & \multirow{4}{*}{$\begin{array}{l}\text { Percepción familiar } \\
\text { de la calidad de los } \\
\text { elementos construc- } \\
\text { tivos }\end{array}$} & Buena & 62,58 & Buena & 62,63 \\
\hline & & & Regular & 28,57 & Regular & 22,73 \\
\hline & & & Mala & 6,80 & Mala & 9,08 \\
\hline & & & NS/NR & 2,05 & NS/NR & 5,56 \\
\hline & \multirow{4}{*}{$\begin{array}{l}\text { Instalaciones y } \\
\text { servicios internos }\end{array}$} & \multirow{4}{*}{$\begin{array}{l}\text { Percepción familiar } \\
\text { de la calidad de las } \\
\text { instalaciones inter- } \\
\text { nas }\end{array}$} & Buena & 81,63 & Buena & 82,32 \\
\hline & & & Regular & 10,88 & Regular & 11,62 \\
\hline & & & Mala & 7,49 & Mala & 3,03 \\
\hline & & & NS/NR & 0,00 & NS/NR & 3,03 \\
\hline & \multirow{6}{*}{$\begin{array}{l}\text { Condiciones de } \\
\text { confort }\end{array}$} & \multirow{3}{*}{$\begin{array}{l}\text { Percepción familiar } \\
\text { de las condiciones } \\
\text { de aislamiento tér- } \\
\text { mico }\end{array}$} & Buena & 59,86 & Buena & 42,93 \\
\hline & & & Regular & 19,73 & Regular & 20,71 \\
\hline & & & Mala & 20,41 & Mala & 36,36 \\
\hline & & \multirow{3}{*}{$\begin{array}{l}\text { Percepción familiar } \\
\text { de las condiciones } \\
\text { de aislamiento acús- } \\
\text { tico }\end{array}$} & Buena & 16,33 & Buena & 24,75 \\
\hline & & & Regular & 19,73 & Regular & 18,18 \\
\hline & & & Mala & 63,94 & Mala & 57,07 \\
\hline \multirow{3}{*}{\multicolumn{2}{|c|}{ Costos y financiamiento }} & $\begin{array}{l}\text { Valor promedio de la } \\
\text { vivienda }\end{array}$ & \multicolumn{2}{|c|}{$\$ 20020000$} & \multicolumn{2}{|c|}{$\$ 19760000$} \\
\hline & & $\begin{array}{l}\text { Valor promedio del } \\
\text { metro cuadrado }\end{array}$ & \multicolumn{2}{|c|}{$\$ 326271$} & \multicolumn{2}{|c|}{$\$ 296875$} \\
\hline & & $\begin{array}{l}\text { Acceso a crédito } \\
\text { bancario }\end{array}$ & \multicolumn{2}{|c|}{$65,31 \%$} & \multicolumn{2}{|c|}{$31,82 \%$} \\
\hline
\end{tabular}

Fuente: elaboración propia

46 Tecnura | Vol. $17 \mid$ No. 38 | Octubre - Diciembre de 2013 


\section{investigación}

Tabla 5. Resultados obtenidos de la Dimensión 2

\begin{tabular}{|c|c|c|c|c|c|c|}
\hline \multicolumn{3}{|c|}{ Dimensión 2. Condiciones comunitarias de habitabilidad } & \multicolumn{2}{|c|}{ Modelo Mercado(\%) } & \multicolumn{2}{|c|}{ Modelo PSHV (\%) } \\
\hline \multirow{8}{*}{\multicolumn{2}{|c|}{ Localización de la comunidad }} & \multirow{4}{*}{$\begin{array}{l}\text { Accesibilidad a acti- } \\
\text { vidades básicas. }\end{array}$} & Buena & 48,98 & Buena & 54,54 \\
\hline & & & Regular & 20,41 & Regular & 11,21 \\
\hline & & & Mala & 7,75 & Mala & 11,52 \\
\hline & & & NS/NR & 22,86 & NS/NR & 22,73 \\
\hline & & \multirow{4}{*}{$\begin{array}{l}\text { Accesibilidad desde } \\
\text { y hacia zonas veci- } \\
\text { nas }\end{array}$} & Buena & 88,78 & Buena & 84,84 \\
\hline & & & Regular & 6,12 & Regular & 8,34 \\
\hline & & & Mala & 3,06 & Mala & 3,79 \\
\hline & & & NS/NR & 2,04 & NS/NR & 3,03 \\
\hline \multirow[t]{28}{*}{ Servicios públicos } & \multirow{4}{*}{$\begin{array}{l}\text { Agua } \\
\text { potable }\end{array}$} & \multirow{4}{*}{$\begin{array}{l}\text { Percepción familiar } \\
\text { de la calidad en la } \\
\text { prestación del ser- } \\
\text { vicio }\end{array}$} & Buena & 81,63 & Buena & 79,79 \\
\hline & & & Regular & 18,37 & Regular & 21,21 \\
\hline & & & Mala & 0,00 & Mala & 0,00 \\
\hline & & & $\mathrm{NS} / \mathrm{NR}$ & 0,00 & NS/NR & 0,00 \\
\hline & \multirow{4}{*}{$\begin{array}{l}\text { Energía } \\
\text { eléctrica }\end{array}$} & \multirow{4}{*}{$\begin{array}{l}\text { Percepción familiar } \\
\text { de la calidad en la } \\
\text { prestación del ser- } \\
\text { vicio }\end{array}$} & Buena & 93,88 & Buena & 93,94 \\
\hline & & & Regular & 6,12 & Regular & 4,55 \\
\hline & & & Mala & 0,00 & Mala & 1,52 \\
\hline & & & NS/NR & 0,00 & NS/NR & 0,00 \\
\hline & \multirow{4}{*}{$\begin{array}{l}\text { Gas natural } \\
\text { domiciliario }\end{array}$} & \multirow{4}{*}{$\begin{array}{l}\text { Percepción familiar } \\
\text { de la calidad en la } \\
\text { prestación del ser- } \\
\text { vicio }\end{array}$} & Buena & 95,92 & Buena & 93,94 \\
\hline & & & Regular & 0,00 & Regular & 3,03 \\
\hline & & & Mala & 0,00 & Mala & 1,52 \\
\hline & & & NS/NR & 4,08 & $\mathrm{NS} / \mathrm{NR}$ & 1,52 \\
\hline & \multirow{4}{*}{$\begin{array}{l}\text { Desagüe } \\
\text { de aguas } \\
\text { negras }\end{array}$} & \multirow{4}{*}{$\begin{array}{l}\text { Percepción familiar } \\
\text { de la calidad en la } \\
\text { prestación del ser- } \\
\text { vicio }\end{array}$} & Buena & 87,76 & Buena & 75,76 \\
\hline & & & Regular & 10,20 & Regular & 15,15 \\
\hline & & & Mala & 2,04 & Mala & 7,58 \\
\hline & & & NS/NR & 0,00 & NS/NR & 1,52 \\
\hline & \multirow{4}{*}{$\begin{array}{l}\text { Desagüe } \\
\text { de aguas } \\
\text { lluvias }\end{array}$} & \multirow{4}{*}{$\begin{array}{l}\text { Percepción familiar } \\
\text { de la calidad en la } \\
\text { prestación del ser- } \\
\text { vicio }\end{array}$} & Buena & 77,55 & Buena & 72,73 \\
\hline & & & Regular & 18,37 & Regular & 24,24 \\
\hline & & & Mala & 4,08 & Mala & 1,52 \\
\hline & & & NS/NR & 0,00 & NS/NR & 1,52 \\
\hline & \multirow{4}{*}{$\begin{array}{l}\text { Alumbrado } \\
\text { público }\end{array}$} & \multirow{4}{*}{$\begin{array}{l}\text { Percepción familiar } \\
\text { de la calidad en la } \\
\text { prestación del ser- } \\
\text { vicio }\end{array}$} & Buena & 75,51 & Buena & 54,55 \\
\hline & & & Regular & 24,49 & Regular & 24,24 \\
\hline & & & Mala & 0,00 & Mala & 21,21 \\
\hline & & & NS/NR & 0,00 & NS/NR & 0,00 \\
\hline & \multirow{4}{*}{$\begin{array}{l}\text { Telefonía } \\
\text { fija }\end{array}$} & \multirow{4}{*}{$\begin{array}{l}\text { Percepción familiar } \\
\text { de la calidad en la } \\
\text { prestación del ser- } \\
\text { vicio }\end{array}$} & Buena & 89,80 & Buena & 93,94 \\
\hline & & & Regular & 2,04 & Regular & 3,03 \\
\hline & & & Mala & 6,12 & Mala & 1,52 \\
\hline & & & NS/NR & 2,04 & NS/NR & 1,52 \\
\hline
\end{tabular}




\section{investigación}

\begin{tabular}{|c|c|c|c|c|c|c|}
\hline \multicolumn{3}{|c|}{ Dimensión 2. Condiciones comunitarias de habitabilidad } & \multicolumn{2}{|c|}{ Modelo Mercado(\%) } & \multicolumn{2}{|c|}{ Modelo PSHV (\%) } \\
\hline & \multirow{4}{*}{$\begin{array}{l}\text { Recolección } \\
\text { de basuras }\end{array}$} & \multirow{4}{*}{$\begin{array}{l}\text { Percepción familiar } \\
\text { de la calidad en la } \\
\text { prestación del ser- } \\
\text { vicio }\end{array}$} & Buena & 91,84 & Buena & 72,73 \\
\hline & & & Regular & 6,12 & Regular & 15,15 \\
\hline & & & Mala & 2,04 & Mala & 12,12 \\
\hline & & & NS/NR & 0,00 & NS/NR & 0,00 \\
\hline \multirow{16}{*}{\multicolumn{2}{|c|}{ Infraestructura vial }} & \multirow{4}{*}{$\begin{array}{l}\text { Percepción familiar } \\
\text { del estado de vías } \\
\text { peatonales internas }\end{array}$} & Buena & 83,67 & Buena & 80,30 \\
\hline & & & Regular & 14,29 & Regular & 13,64 \\
\hline & & & Mala & 2,04 & Mala & 3,03 \\
\hline & & & NS/NR & 0,00 & NS/NR & 3,03 \\
\hline & & \multirow{4}{*}{$\begin{array}{l}\text { Percepción familiar } \\
\text { del estado de vías } \\
\text { peatonales internas }\end{array}$} & Buena & 83,67 & Buena & 83,33 \\
\hline & & & Regular & 14,29 & Regular & 10,61 \\
\hline & & & Mala & 2,04 & Mala & 3,03 \\
\hline & & & NS/NR & 0,00 & NS/NR & 3,03 \\
\hline & & \multirow{4}{*}{$\begin{array}{l}\text { Percepción familiar } \\
\text { del estado de vías } \\
\text { peatonales barriales }\end{array}$} & Buena & 89,80 & Buena & 74,24 \\
\hline & & & Regular & 10,20 & Regular & 16,67 \\
\hline & & & Mala & 0,00 & Mala & 6,06 \\
\hline & & & NS/NR & 0,00 & NS/NR & 3,03 \\
\hline & & \multirow{4}{*}{$\begin{array}{l}\text { Percepción familiar } \\
\text { del estado de vías } \\
\text { vehiculares barriales }\end{array}$} & Buena & 89,80 & Buena & 78,79 \\
\hline & & & Regular & 10,20 & Regular & 10,61 \\
\hline & & & Mala & 0,00 & Mala & 7,58 \\
\hline & & & NS/NR & 0,00 & NS/NR & 3,03 \\
\hline \multirow{5}{*}{\multicolumn{2}{|c|}{ Desarrollo urbanístico }} & $\begin{array}{l}\text { Cumplimiento } \\
\text { de normativa urbana }\end{array}$ & \multicolumn{2}{|l|}{ Cumple } & \multicolumn{2}{|l|}{ Cumple } \\
\hline & & Tipo de loteo & \multicolumn{4}{|c|}{ Similar en ambos modelos } \\
\hline & & $\begin{array}{l}\text { Implantación } \\
\text { de las viviendas }\end{array}$ & \multicolumn{4}{|c|}{ Similar en ambos modelos } \\
\hline & & Índice de ocupación & \multicolumn{2}{|l|}{$65,00 \%$} & \multicolumn{2}{|l|}{$65,00 \%$} \\
\hline & & $\begin{array}{l}\text { Densidad de } \\
\text { vivienda }\end{array}$ & \multicolumn{2}{|c|}{$270,82 \mathrm{viv} / \mathrm{Ha}$} & \multicolumn{2}{|c|}{$203,24 \mathrm{viv} / \mathrm{Ha}$} \\
\hline \multirow{3}{*}{\multicolumn{2}{|c|}{ Servicios sociales }} & Seguridad & \multicolumn{2}{|c|}{ Bueno: $37,39 \%$} & \multicolumn{2}{|c|}{ Bueno: $37,39 \%$} \\
\hline & & Salud & \multicolumn{4}{|c|}{ Aún no existen centros de salud } \\
\hline & & Educación & \multicolumn{4}{|c|}{$\begin{array}{l}\text { Utilización: } 66,96 \% \\
\text { Bueno: } 77,92 \%\end{array}$} \\
\hline
\end{tabular}

Fuente: elaboración propia 


\section{investigación}

Tabla 6. Resultados obtenidos de la Dimensión 3

\begin{tabular}{|c|c|c|}
\hline \multicolumn{2}{|c|}{ Dimensión 3. Organización y autogestión comunitaria } & Modelo PSHV \\
\hline \multirow[t]{2}{*}{ Desarrollo organizacional } & $\begin{array}{l}\text { Características de la orga- } \\
\text { nización }\end{array}$ & $\begin{array}{l}\text { En todos los casos se generó una organización formal; } \\
\text { sin embargo, en dos proyectos (Nueva Ciudad Etapa } \\
1 \text { y Casa Grande Etapa 1), esta decisión se tomó des- } \\
\text { pués de que el proyecto ya estaba en marcha }\end{array}$ \\
\hline & $\begin{array}{l}\text { Definición de objetivos co- } \\
\text { munes }\end{array}$ & $\begin{array}{l}\text { Solo en uno de los proyectos (Renacer) se generaron } \\
\text { procesos colectivos para toma de decisiones. En los } \\
\text { otros, estas decisiones eran presentadas a las familias } \\
\text { vinculadas por los líderes de la organización }\end{array}$ \\
\hline \multirow[t]{2}{*}{ Desempeño organizacional } & Relación con actores & $\begin{array}{l}\text { Solo en el caso del Proyecto Renacer, esta dinámica } \\
\text { fue planteada para la solución del problema habitacio- } \\
\text { nal. En los otros casos simplemente cada familia acudió } \\
\text { a la ONG oferente }\end{array}$ \\
\hline & $\begin{array}{l}\text { Aporte organizativo al pro- } \\
\text { yecto }\end{array}$ & $\begin{array}{l}\text { Aunque en el Proyecto Renacer, se generó una mayor } \\
\text { dinámica comunitaria, aún en este caso fue muy pobre, } \\
\text { porque la mayoría de las funciones estaban a cargo de } \\
\text { los líderes, sin consultar a la base. No hubo participa- } \\
\text { ción ni en el diseño ni en la construcción }\end{array}$ \\
\hline
\end{tabular}

Fuente: elaboración propia

no se diferencian sustancialmente del modelo de mercado; debido a que las ONG encargadas de los proyectos no promovieron diálogos, negociaciones y consensos sobre los procesos y los productos, sino que optaron por presentar decisiones definidas y manejar un esquema de demanda. Una de las principales características del proceso de PSHV, que es el fortalecimiento de las capacidades sociales de las comunidades, no fue tenido en cuenta en los proyectos analizados.

Debido a que el proyecto Renacer fue concebido como solución al problema habitacional de un grupo de familias por causa de un deslizamiento de tierra donde residía la mayor parte de los habitantes, este sí permitió que operaran como un colectivo organizado en búsqueda de un hábitat para reubicarse y no como individuos buscando unidades familiares aisladas. En este caso, el proceso de PSHV se adaptó con relativa facilidad a las condiciones de una demanda comunitaria previamente organizada.

En cuanto al valor global de la vivienda, no existen diferencias importantes; pero si se considera el valor por metro cuadrado, este es menor en las soluciones ofrecidas por PSHV, debido a la mayor área de la vivienda. Existe una diferencia importante en los dos modelos en cuanto a las fuentes de financiación utilizadas. En la opción de mercado es más común usar el crédito ofrecido por el sistema bancario y en el modelo PSHV se contemplan otras fuentes como: ahorros y crédito cooperativo, entre otras. En ambos casos, las organizaciones oferentes aseguran a los compradores la titulación de la propiedad. 


\section{CONCLUSIONES}

No se observan diferencias sustanciales en los resultados-productos; con excepción de una mayor área de lote en las soluciones de PSHV y mejor proporcionalidad en sus dimensiones. Estas diferencias conducen a que los ocupantes de viviendas ofrecidas por el mercado realicen ampliaciones en altura, mientras que los ocupantes de viviendas PSHV las hagan en la planta. En este punto - opción de ampliación-, se observa una evolución en la oferta de la modalidad de mercado, que era exclusiva de ofertas de PSHV.

Aunque formalmente las acciones realizadas en los procesos de PSHV se ajustaron a sus principios, en la realidad, se dejaron en un plano secundario los principios de solidaridad, participación, cooperación, autogestión y democracia, que debían regir la construcción de las soluciones habitacionales en esta modalidad.

En términos de las instalaciones internas de las viviendas, no se identifican diferencias significativas en los dos modelos.

En cuanto a las condiciones urbanísticas, se pudo apreciar que los proyectos generados por PSHV tienen una densidad de vivienda menor que los ofrecidos por el mercado; pero en sus otras características ofrecen condiciones similares.

Los dos modelos de producción de VIS han avanzado en la apropiación del concepto de hábitat; las viviendas han sido concebidas como entornos habitacionales y no como estructuras arquitectónicas aisladas.

Este acercamiento en las condiciones del producto ofrecido ha sido promovido por la misma dinámica del mercado, al tratar de ofrecer un producto que sea aceptado por los grupos de más bajos ingresos.

Algunos interrogantes que quedan después de este análisis y que se proponen para futuros estudios son: ¿La convergencia de los modelos de producción de vivienda, mediante mercado y PSHV, observada en proyectos habitacionales de grupos de bajos recursos, se presenta en el conjunto de la ciudad?, si es así, ¿se limita a la ciudad de Bogotá, o se extiende a otras capitales o ciudades intermedias?, ¿cómo se está dando este proceso en otros centros urbanos del país?, ¿se debe esperar que el mercado vaya adaptando paulatinamente su respuesta a la demanda de los grupos de bajos ingresos?, ¿será cierto que el mercado es el llamado a resolver definitivamente el problema de vivienda en las ciudades colombianas?, ¿cómo pueden las organizaciones encargadas de manejar procesos de PSHV, mejorar efectivamente las capacidades sociales de las comunidades y entregar no solo vivienda, sino hábitat?

\section{FINANCIAMIENTO}

Este proyecto fue financiado por SWISSCONTACT Colombia, Fundación Suiza de Cooperación para el Desarrollo Técnico.

\section{AGRADECIMIENTOS}

Los autores agradecen al Proyecto Curricular de Ingeniería Civil de la Universidad Distrital Francisco José de Caldas y a Departamento de Ingeniería Civil de la Universidad de La Salle. 


\section{REFERENCIAS}

[1] Fundación Suiza de Cooperación para el desarrollo técnico SWISSCONTACT, Análisis de modelos para producción de vivienda de interés social en Bogotá, SWISSCONTACT: Bogotá, 2012.

[2] I. Aguado, C. Echebarría y J. Barrutia, "El desarrollo sostenible a lo largo de la historia del pensamiento económico", Revista de Economía Mundial, Vol. 21, No. 87, pp $110,2009$.

[3] M. Max-Neef, A. Elizalde y M. Hopenhavn, Desarrollo a Escala Humana: una opción para el futuro. Medellín: Cepaur y Fundación Dag Hammarskjöld, 1997.

[4] A. Sen, Desarrollo y Libertad. Bogotá: Editorial Planeta, 2000.

[5] Habitat International Coalition (HIC), De la marginación a la ciudadanía: 38 casos de producción y gestión social el hábitat, Barcelona: Habitat International Coalition, 2004.

[6] Declaración Universal de los Derechos Humanos, [Adoptada y proclamada por la Asamblea General de Naciones Unidas], Resolución 217 A (III), de 10 de diciembre de 1948, Paris.

[7] Naciones Unidas, Consejo Económico y Social, Los derechos económicos, sociales y culturales: Informe del Relator Especial sobre una vivienda adecuada, como parte del derecho a un nivel de vida adecuado, Sr. Miloon Kothari, Marzo de 2004. E/ CN.4/2004/48.
[8] F. Giraldo y J. Torres, Hábitat y Desarrollo Humano, Cuadernos PNUD, UN-Hábitat, Bogotá, 2004.

[9] E. Leff, Saber ambiental: sustentabilidad, racionalidad, complejidad, poder. México: Siglo XXI, 1998.

[10] E. Ortiz, Notas sobre la producción social de vivienda. Elementos básicos para su conceptualización e impulso, México: Casa y Ciudad, 2004.

[11] FEDEVIVIENDA, (2012, dic.) "Producción Social de Hábitat”, [en línea]. Disponible en: http://www.fedevivienda.org.co/ $\mathrm{psh} /$.

[12] Departamento Nacional de Estadística DANE. (2012, Nov.), "Déficit de Vivienda 2005" [en línea]. Disponible en http://www.dane.gov.co/index.php?option=com_content\&view $=$ article $\&$ id $=473 \&$ Itemid $=66$.

[13] G. Romero, La producción social del Hábitat; reflexiones sobre su historia, concepciones y propuestas, México: CYTEDHABYTED-Red XIV, 2004.

[14] E. Ortiz, "La producción social del hábitat: ¿opción marginal o estrategia transformadora?", Dfensor, Vol. 7, No. 11, pp. 10-16, noviembre, 2009.

[15] N. Jeifetz y M. Rodríguez, (2012, Nov.), "El movimiento cooperativista autogestionario en Buenos Aires y la construcción de las politicas de hábitat popu- 
lar”, TRIALOG Journal, 78, [en línea]. Disponible en http:/www.trialog-journal.de/.

[16] E. Greenwood, Metodología de la investigación social. Buenos Aires: Editorial Paidos, 1973.
[17] M. Rodríguez, C. Buthet, J. Scavuzzo, y A. Taborda, Indicadores de resultados e impactos. metodología de aplicación en proyectos participativos de hábitat popular, Argentina: Agencia Alemana para el Desarrollo (MISEREOR), Coalición Internacional del Hábitat (HIC-AL), 2004. 